\title{
Assembly \& Structure of CFA/I Fimbriae from Enterotoxigenic Escherichia coli
}

\author{
Di Xia * Stephen J. Savarino **, Esther Bullitt*** \\ * National Cancer Institute, NIH, Bethesda MD \\ ** Naval Medical Research Center, Silver Spring MD \\ *** Boston University School of Medicine, Boston MA
}

Enterotoxigenic Escherichia coli (ETEC) bacteria that cause traveler's diarrhea utilize pili to initiate infection via pilus binding to epithelial cells in the small intestines. According to the World Health Organization, ETEC cause the largest number of recorded community-acquired cases of childhood diarrhea in the developing world, and are the most common cause of traveler's diarrhea. Through a multi-disciplinary approach including x-ray crystallography, electron microscopy, site-directed mutagenesis, and genetic sequence analysis we elucidate the assembly and structure of CFA/I pili expressed on ETEC.

We show that the distinction between Class I pili from the chaperone/usher pathway (e.g. P-pili from uropathogenic bacteria) and Class 5 pili from the alternate chaperone pathway (e.g. CFA/I pili), which was based on the lack of genetic sequence homology, does not correlate with any major structural or functional differences between classes. Pilin subunits transit the outer membrane through an usher that can accommodate single subunits, but not the assembled helical filament. We identify a proline residue in the major pilin, $\mathrm{CfaB}$, that appears to isomerize from the trans to cis conformation, producing the conformational change required for assembly of the mature pilus filament comprising about 1,000 subunits. Mutation of this proline to a phenylalanine results in a loss of hemagglutination over time, suggesting that proper assembly of these fimbriae is essential for sustained bacterial binding to the host cell.

Analysis of genetic variability among clinical strains representative of the eight discrete Class 5 fimbrial subtypes, in combination with structural data, shows that each bacterial strain presents a distinct outer surface of $\mathrm{CfaB}$, while the interior and protein-protein interface residues are more highly conserved. These data suggest that protein surface variability facilitates evasion of the immune system by ETEC. 


\section{References:}

Li YF, Poole S, Nishio K, Jang K, Rasulova F, McVeigh A, Savarino SJ, Xia D, \& Bullitt E (2009) Structure of CFA/I fimbriae from enterotoxigenic Escherichia coli.

Proc Natl Acad Sci USA 106 (26) :10793-10798.

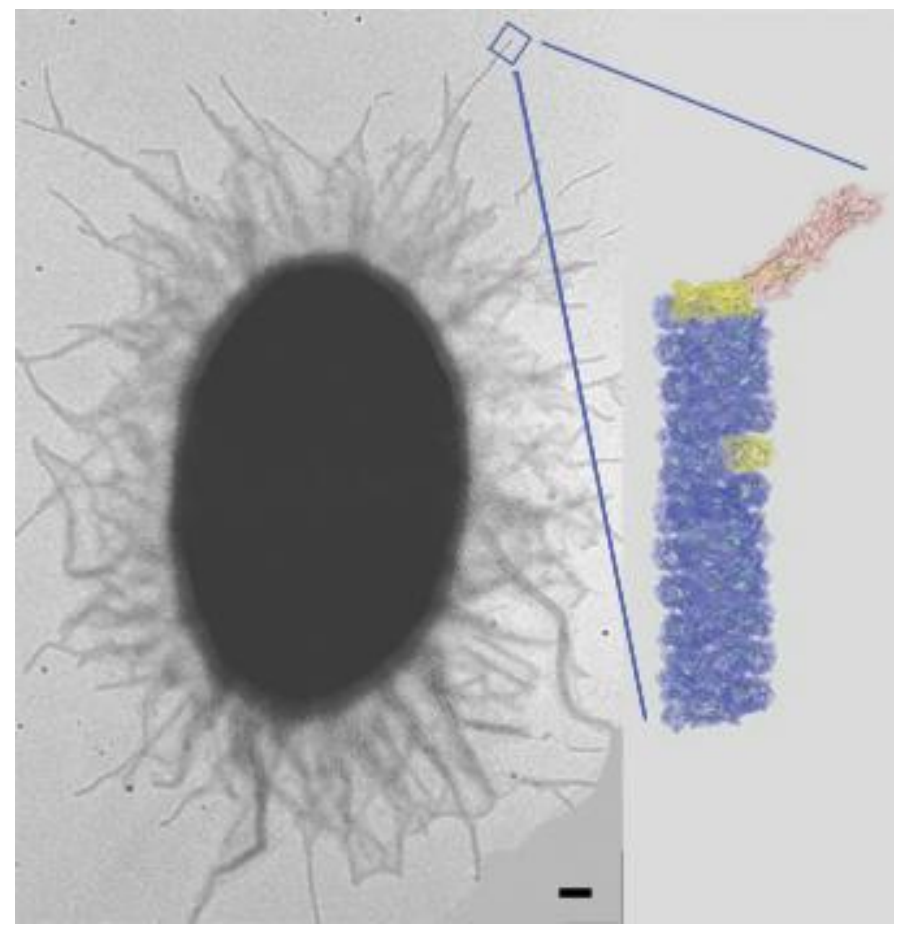

ETEC bacteria expressing CFA/I pili as a critical virulence factor (left). A model of the CFA/I pilus structure from hybrid analysis of EM, x-ray, and microbiological data shows the tip adhesin extending out from the helical filament. Mag. bar $0.5 \mu \mathrm{m}$. 